\title{
779 INHIBITING TYPE-I INTERFERON SIGNALING PROMOTES MEMORY T-CELL FORMATION FOLLOWING IMMUNIZATION WITH LISTERIA ANTI-CANCER VACCINES
}

Zachary Morrow*, John-Demian Sauer. The University of Wisconsin-Madison, Madison, WI, United States

Background The aspiration of cancer immunotherapy is to generate large numbers of highly functional anti-tumor CD8+ T-cells. We and others have optimized Listeria monocytogenes as a powerful anti-cancer vaccine platform to drive such T-cell responses. Early clinical trial data suggest the number of Tcells generated correlates with efficacy, demanding an understanding of the factors that dictate vaccine-induced T-cell responses. The CD8 + T-cell response is intimately linked to magnitude and quality of the innate immune response triggered by vaccines. Listeria-based vaccines activate numerous innate pathways and can be engineered to hyper- or hypoinduce these pathways. We sought to understand how modulating innate immunity would impact vaccine efficacy.

Methods To dissect the impact of type I interferon signaling and the inflammasomes on L. monocytogenes induced T-cell responses, we immunized IFNAR-/-, Caspase1/11-/-, and novel IFNAR-/-Caspase1/11-/- double knockouts mice we generated for this study. CD8 + T-cell responses were assessed at the peak T-cell response, after contraction and memory formation, and after rechallenge. The phenotype and magnitude of CD8 + T-cells was assessed at each stage, and functional outcomes were assessed by measuring protection from reinfection by wild-type Listeria.

Results IFNAR-/- mice developed the largest number of CD8+ T-cells during the peak primary response contradicting the dogma that Type-I Interferon promotes robust CD8 + T-cell responses. Caspase1/11-/- mice were not significantly different from wild-type mice. The frequency of short-lived effector cells (assessed by expression of CD127 and KLRG1) was no different between wild-type and IFNAR-/- mice, however we observed more than twice as many memory precursor cells at the peak CD8 + T-cell response. These findings extend to the memory and recall stage with more antigen-specific T-cells observed after contraction and upon rechallenge. Finally, IFNAR-/- mice are remarkably more protected from wild-type Listeria rechallenge than their counterparts after immunization demonstrating the efficacy of the increased memory T-cell pool. Data are representative of at least two independent replicates with at least 5 mice per group and significance was assessed by one-way ANOVA with *p $<0.05$.

Conclusions We demonstrated that type-I interferon signaling deficiency leads to enhanced prophylactic vaccine efficacy through increased memory T-cell formation. Ultimately, for patients with slow growing tumors or with high-risk mutations, prophylactic tumor vaccines could elicit life-long protection from disease. Importantly, increased memory precursor Tcell abundance did not come at the expense of short-lived effectors leaving open the possibility that blocking Type-I IFN could potentiate lasting immunological memory in both the therapeutic and prophylactic setting.

http://dx.doi.org/10.1136/jitc-2021-SITC2021.779 